\title{
Control of spontaneous ovarian tumors by CD8+ T cells through NKG2D-targeted delivery of antigenic peptide
}

Tae Heung Kang ${ }^{1}$, Jayne Knoff ${ }^{2}$, Benjamin Yang ${ }^{2}$, Ya-Chea Tsai ${ }^{2}$, Liangmei He ${ }^{2}$, Chien-Fu Hung ${ }^{2,4}$ and T-C Wu ${ }^{2,3,4,5^{*}}$

\begin{abstract}
Background: There is an urgent need to develop targeted therapies for the control of advanced stage ovarian cancer because it is the most deadly gynecologic cancer. Antigen-specific immunotherapy is a promising approach because of the potential of the immune system to specifically target tumors without the toxicity associated with traditional chemoradiation. However, one of the major limitations for antigen-specific cancer immunotherapy is the pre-existing immune tolerance against endogenous targeted tumor antigens that frequently evolves during carcinogenesis. Here, we described the creation of a therapeutic agent comprised of a tumor-homing module fused to a functional domain capable of selectively rendering tumor cells sensitive to foreign antigen-specific CD8+ T cell-mediated immune attack, thereby circumventing many aspects of immune tolerance. The tumor-homing module, NKG2D, specifically binds to NKG2D ligand that is commonly overexpressed in ovarian tumors. The functional domain is comprised of the Fc portion of IgG2a protein and foreign immunogenic CD $8^{+}$ $T$ cell epitope flanked by furin cleavage sites $(R)$, which can be recognized and cleaved by furin that is highly expressed in the tumor microenvironment.

Results: We show that this therapeutic chimeric protein specifically loaded antigenic epitope onto the surface of NKG2D ligand-expressing ovarian tumor cells, rendering ovarian tumors susceptible to antigen-specific CTL-mediated killing in vitro. Furthermore, we show that intraperitoneal administration of our therapeutic chimeric protein followed by adoptive transfer of antigen-specific CD8+ T cells generates potent antitumor effects and significant accumulation of antigen-specific CD8+ T cells in the tumor loci.
\end{abstract}

Conclusions: Our findings have promise for bypassing immune tolerance to enhance cancer immunotherapy.

Keywords: NKG2D, Ovarian cancer, Immunotherapy, T cell adoptive transfer, TgMISIIR-TAg transgenic mice

\section{Background}

Advanced ovarian cancer is one of the most deadly malignancies and is responsible for the highest mortality rate among patients with cancers of the female reproductive system in the United States. Existing therapies for ovarian cancer, such as surgery and chemotherapy, have significant side effects and rarely result in long-term cures for patients with locally advanced or metastatic disease, although they can provide remission for several years [1,2]. The lack of

\footnotetext{
* Correspondence: wutc@jhmi.edu

${ }^{2}$ Department of Pathology, School of Medicine, Johns Hopkins University, Bldg. CRBII Rm. 309, 1550 Orleans Street, Baltimore, Maryland 21231, USA ${ }^{3}$ Department of Obstetrics and Gynecology, Johns Hopkins University, Baltimore, MD 21205, USA

Full list of author information is available at the end of the article
}

curative treatments and high proportion of patients diagnosed with advanced disease underscores the urgent need to develop innovative and targeted therapies for the control of advanced stage ovarian cancer. Antigen-specific immunotherapy is a promising treatment strategy to enhance the control of advanced ovarian cancer, particularly to target minimal residual disease. Antigen-specific immunotherapy has the ability to harness the immune system to specifically target tumors without the toxicity associated with traditional chemoradiation (for review, see [3]). However, one of the major limitations for antigen-specific cancer immunotherapy is the inherited immune tolerance against endogenous tumor antigens targeted by the antigen-specific immunotherapy. 
We have previously demonstrated that by selectively coating tumor cells with immunodominant foreign immunogenic CD8+ $\mathrm{T}$ cell peptide(s) recognized by preexisting immunity, immune tolerance can be bypassed and pre-existing antigen-specific immunity was able to control the tumor [4]. We previously used a murine ovarian cancer model to design a therapeutic chimeric protein containing a tumor-homing module fused to a functional cargo domain. The tumor-homing portion of the therapeutic chimeric protein, an anti-mesothelin single chain variable fragment (meso-scFv), had high binding affinity to mesothelin [4], which is overexpressed by most ovarian cancers [5,6]. The cargo domain of the therapeutic chimeric protein was comprised of the Fc portion of the IgG2a protein and a MHC class I-restricted foreign immunogenic CTL epitope, OVA, flanked by furin cleavage sites. We showed that the antigenic CTL epitope could be loaded onto tumor cells following selective cleavage of the amino acid sequence RVKR by furin. Many tumors, including ovarian tumors (for review, see [7]), highly express furin on the cell surface and in the extracellular space [8-12]. We showed that meso-scFv preferentially delivered OVA to mesothelin-expressing ovarian cancer cells, where cleavage by furin released the foreign immunogenic CTL epitope to be loaded on MHC class I molecules of tumor cells [4]. Notably, this rendered tumor cells susceptible to OVA-specific CTL-mediated killing, both in vitro and in vivo [4]. Thus, specific molecules expressed by ovarian tumors, such as mesothelin, can be used as targets for the delivery of antigeneic CTL peptides. The encouraging results from these studies in the ovarian tumor model warrant further exploration of the molecular targets specifically expressed by tumors for our therapeutic approach.

The identification of surface molecules that are specifically expressed in tumor cells but not in normal cells becomes important to facilitate the specific delivery of antigenic CTL peptides to tumor loci. For example, multiple NKG2D ligands have been identified and are known to be upregulated in transformed, infected, and/or stressed cells but not in substantial amounts in healthy adult cells [13]. The upregulation of NKG2D ligands may be due to cellular and genotoxic stresses, such as excessive proliferation, heat shock, or oxidative stress. Consequently, NKG2D ligands are highly expressed in various tumors of different origins to varying degrees [14]. Thus, protein or antigenic peptides potentially can be linked to NKG2D for their specific delivery to tumor loci. We have previously successfully employed NKG2D to specifically deliver a linked molecule of interest to tumor loci [15].

In the current study, we created a chimeric protein consisting of NKG2D linked to the Fc domain of IgG2a and the OVA CTL peptide flanked by a furin cleavage site to form NKG2D-Fc-RO. We showed that ovarian tumor cells that express the NKG2D ligand Rae-1 can be bound by the chimeric NKG2D-Fc-RO protein and present the OVA CTL peptide through MHC class I molecules. In addition, binding of NKG2D-Fc-RO to tumor cells renders them susceptible to OVA-specific CD8+ T cell-mediated killing. More importantly, we showed that intraperitoneal injection of the chimeric NKG2D-Fc-RO protein is capable of generating potent therapeutic antitumor effects against NKG2D ligand expressing spontaneous ovarian tumor following adoptive transfer of OVA-specific CD8+ T cells. This study serves as a foundation for future therapies utilizing targeted coating of tumor cells with antigenic peptide.

\section{Results}

\section{Generation and characterization of the NKG2D-Fc-RO chimeric protein}

We created a chimeric protein consisting of NKG2D conjugated with Fc (IgG2a) protein and containing OVA peptide $(\mathrm{O})$ flanked by a furin cleavage site (R) (NKG2DFc-RO). Figure 1A shows the schematic diagram of the chimeric NKG2D-Fc-RO construct as well as the control NKG2D-Fc protein without OVA peptide and furin cleavage sites. We have successfully isolated the NKG2DFc-RO and NKG2D-Fc proteins from the supernatant of furin-deficient $\mathrm{CHO}$-based cell line FD11 stably transduced with the expression vector and used SDS-PAGE to assess their size and purity, as shown in Figure 1B. The stained gel shows bands of high purity and of the expected size for both proteins. Because the difference in molecular weight between NKG2D-Fc-RO and NKG2D-Fc is only 12 amino acids, the proteins show similar migration on the SDS-PAGE gel.

\section{The NKG2D-Fc protein binds to murine ovarian tumor cells expressing murine NKG2D ligands}

To determine if the purified NKG2D-Fc-RO protein could bind to murine NKG2D ligand-expressing tumor cells, we selected a Rae-1 (a murine NKG2D ligand)expressing ovarian tumor cell line (MOVCAR) and one Rae-1-negative tumor cell line (B16F10) for our studies (Figure 2A). As shown in Figure 2B, both NKG2DFc-RO and NKG2D-Fc bound to the NKG2D ligandexpressing murine ovarian tumor cells, MOVCAR, but not to the Rae-1-negative B16F10 tumor cells. In comparison, the MOVCAR cells incubated with purified Fc protein alone did not demonstrate specific binding (Figure 2B). This is consistent with previous reports that cells engineered to express the extracellular domain of murine NKG2D are capable of recognizing and binding to cells NKG2D ligand-expressing cells, including Rae1-expressing cells [16]. Taken together, these data indicate that NKG2D-Fc and NKG2D-Fc-RO are able to bind to NKG2D ligand-expressing tumor cells. 


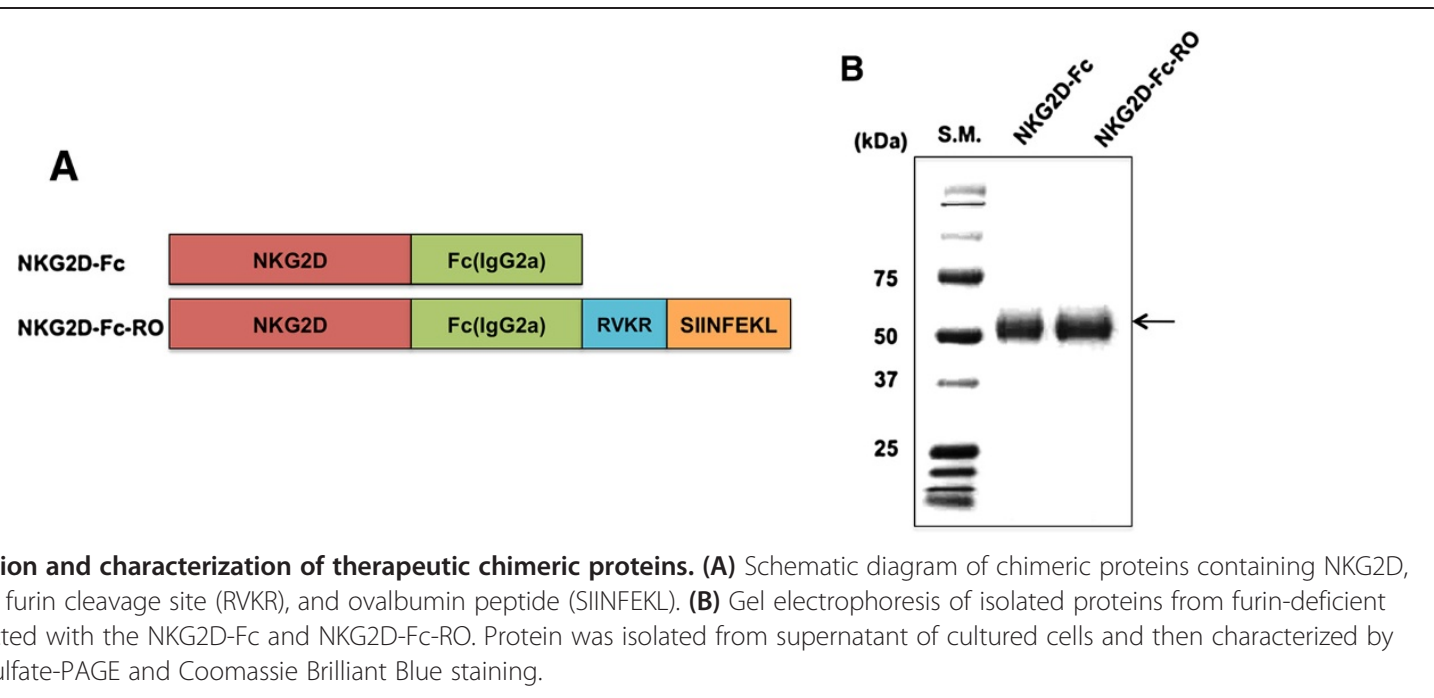

The binding of NKG2D-Fc-RO to NKG2D ligand-expressing tumor cells leads to MHC class I presentation of OVA peptide to OVA-specific $\mathrm{CD}^{+} \mathrm{T}$ cells

We further determined if the binding of NKG2D-Fc-RO to MOVCAR cells would enable MHC class I presentation of OVA peptide to OVA-specific CD8+ T cells and result in the activation of OVA-specific $\mathrm{CD}^{+} \mathrm{T}$ cells. As shown in Figure 3, MOVCAR cells incubated with NKG2DFc-RO led to the greatest activation of OVA-specific CD8 ${ }^{+}$ $\mathrm{T}$ cells compared to MOVCAR cells incubated with NKG2D-Fc. Thus, these results suggest that NKG2DFC-RO specifically binds MOVCAR and facilitates MHC class I presentation of OVA peptide to activate OVAspecific CD8+ T cells.

The binding of NKG2D-Fc-RO to NKG2D ligand-expressing tumor cells renders tumor cells susceptible to

OVA-specific $\mathrm{CD}^{+} \mathrm{T}$ cell-mediated killing

We then determined if binding of NKG2D-Fc-RO to MOVCAR tumor cells would render luciferase-expressing MOVCAR tumor cells susceptible to OVA-specific $\mathrm{CD}^{+} \mathrm{T}$ cell killing. As shown in Figure 4, in the presence of OVAspecific CD8+ T cells, OT-1 cells, luciferase-expressing MOVCAR incubated with NKG2D-Fc-RO had the greatest
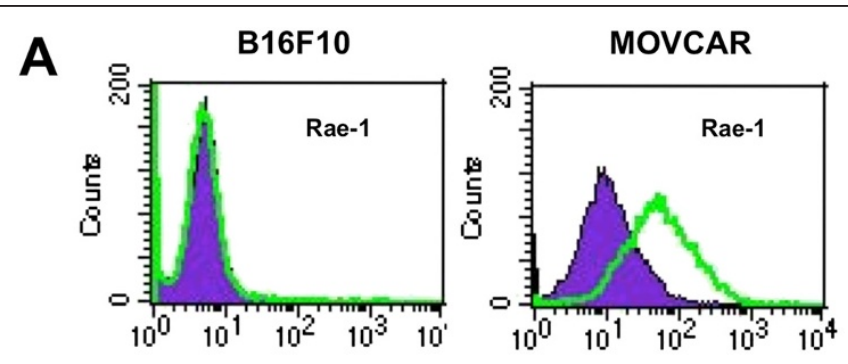

B
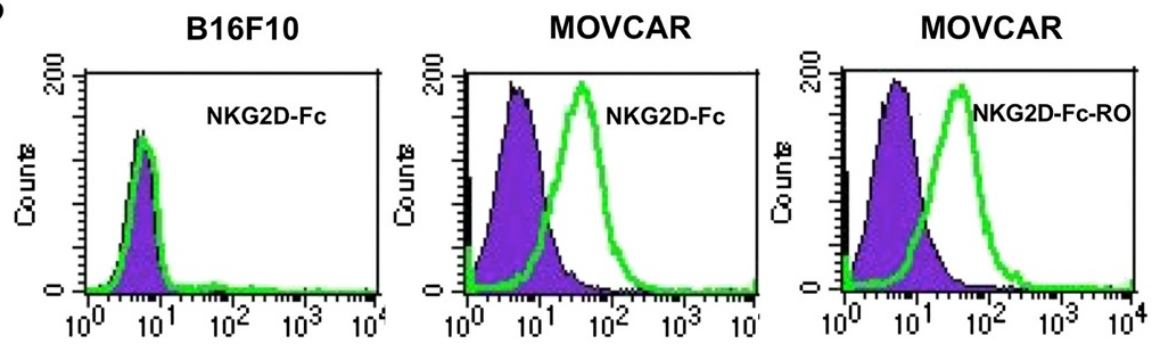

Figure 2 Characterization of the binding of NKG2D-Fc and NKG2D-Fc-RO to NKG2D ligand expressed by MOVCAR tumor cells using flow cytometry. (A) Representative flow cytometry analysis of antibody bound to Rae-1 (clear histogram) or isotype (shaded histogram) with B16F10 (negative control) and MOVCAR cells. (B) Purified proteins FC (shaded histogram), NKG2D-Fc (clear histogram) and NKG2D-Fc-RO (clear histogram) were incubated with either B16F10 or MOVCAR cells and then stained with phycoerythrin (PE)-labeled antimouse Fc antibody. 


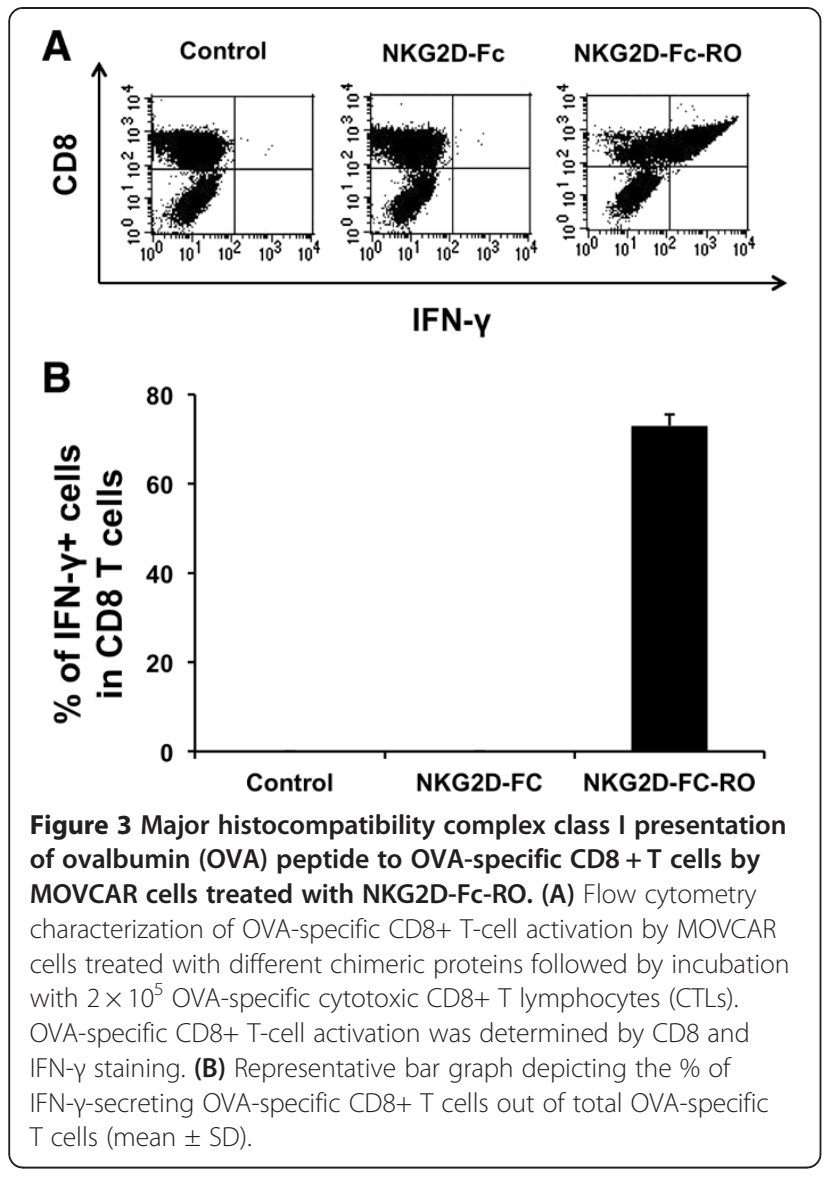

amount of OVA-specific CTL-mediated tumor cell death, as measured by a significant reduction in luminescence activity among different experimental groups. Luciferase-expressing MOVCAR tumor cells incubated with NKG2D-FC did not render tumor cells more susceptible to OVA-specific CD8+ $\mathrm{T}$ cell mediated killing. These data suggest that the binding of NKG2D-Fc-RO to tumor cells renders bound tumor cells susceptible to OVA-specific CTL killing.

\section{Treatment of spontaneous tumor-bearing MISIIR mice with chimeric protein NKG2D-FcRO leads to potent antitumor effects}

We next examined the efficacy of treatment with the chimeric protein including the OVA antigen, NKG2DFc-RO, in tumor-bearing TgMISIIR-TAg transgenic mice. We have previously shown that MISIIR transgenic mice spontaneously develop ovarian tumor approximately 10 weeks after birth [17]. Mice with similar sizes of ovarian tumors were selected to receive treatment with NKG2D-Fc or NKG2D-Fc-RO and OVA-specific OT-1 CD8+ $\mathrm{T}$ cells injected intraperitoneally, as outlined in the treatment schedule depicted in Figure 5A. We found that female TgMISIIR-TAg transgenic mice treated with NKG2D-Fc-RO had significantly reduced tumor mass after 30 days compared to those treated with NKG2D-Fc

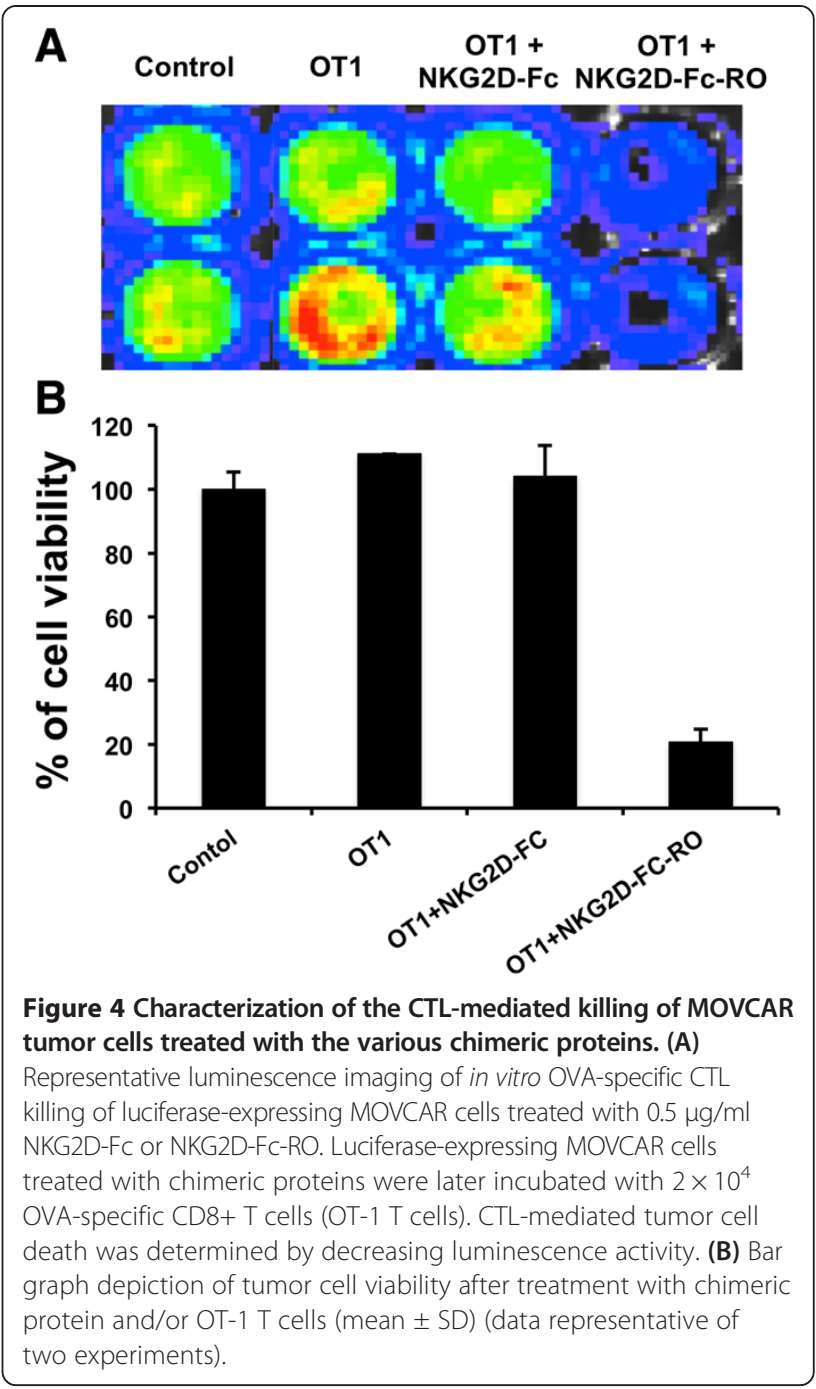

(Figure $5 \mathrm{~B}$ and $\mathrm{C}$ ). These data suggest that intraperitoneal injection of the chimeric NKG2D-Fc-RO protein is capable of generating potent therapeutic antitumor effects against NKG2D ligand expressing ovarian tumors following adoptive transfer of OVA-specific CD8+ T cells.

Spontaneous ovarian tumor-bearing TgMISIIR-TAg transgenic mice treated with chimeric NKG2D-Fc-RO protein in conjunction with adoptive transfer of OVA-specific CD8+ T cells leads to accumulation of OVA-specific CD8+ T cell in the tumor loci In order to determine if treatment of the spontaneous ovarian tumor-bearing mice with the chimeric NKG2DFc-RO protein in conjunction with adoptive transfer of OVA-specific CD8+ $\mathrm{T}$ cell can lead to the expansion of OVA-specific CD8+ T cells in the ovarian tumor loci, we characterized the presence of OVA-specific CD8+ T cell among the TILs derived from the ovarian tumors using OVA CTL peptide loaded $\mathrm{H}-2 \mathrm{~Kb}$ tetramer labeling. As shown in Figure 6A and B, we observed that tumor-bearing 


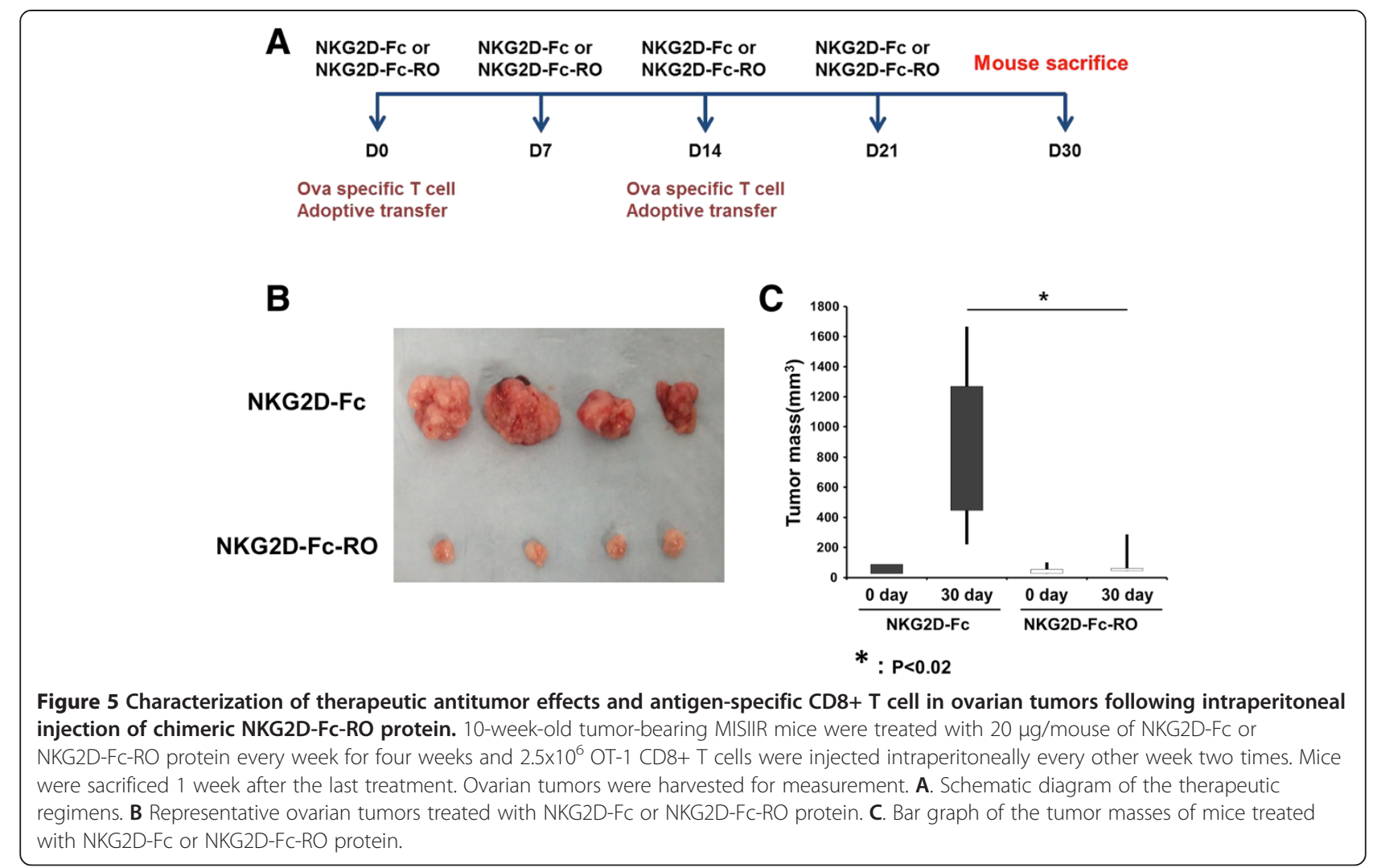

TgMISIIR-TAg transgenic mice treated with NKG2DFc-RO had a significantly higher percentage of OVAspecific CD8+ T cells among TILs compared to those treated with NKG2D-Fc. These data indicate that treatment with NKG2D-Fc-RO may target the linked antigenic peptide to NKG2D ligand expressing tumor cells and promote the presentation of antigenic peptide by tumor cells, which enhances the expansion of OVA-specific CD8+ T cells in the tumor loci.

\section{Discussion}

In this study, we demonstrated that a chimeric protein, NKG2D-Fc-RO, was able to bind to murine NKG2D ligand-expressing ovarian tumor cells in vitro. Furthermore, we were able to use the NKG2D-Fc protein to deliver a model ovalbumin antigenic peptide to NKG2D ligand-expressing ovarian tumor cells to facilitate MHC class I presentation of the antigenic peptide. Additionally, we demonstrated that OVA CTL peptide delivered by NKG2D-Fc-RO to tumor cells rendered tumor cells susceptible to OVA-specific CTL-mediated killing. More importantly, we demonstrated that intraperitoneal injection of the chimeric NKG2D-Fc-RO protein in spontaneous ovarian tumor bearing mice significantly expanded OVAspecific CD8+ $\mathrm{T}$ cells at the tumor loci and resulted in the control of spontaneous ovarian tumors. Thus, our study demonstrated that chimeric proteins containing NKG2D
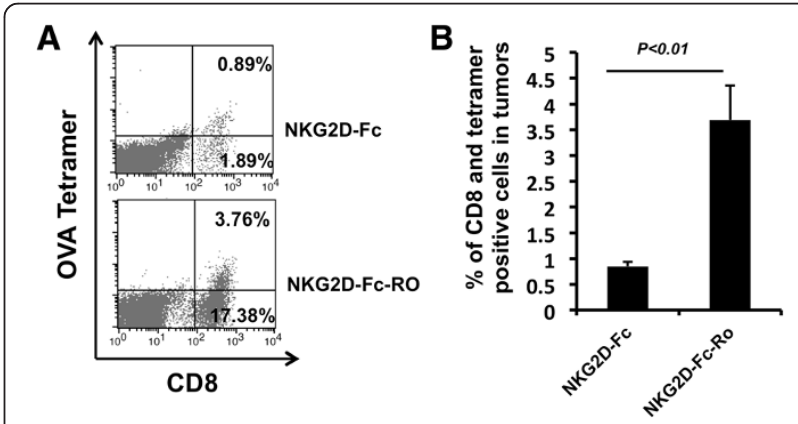

Figure 6 Characterization of OVA-antigen specific CD8+ T cell in the tumor infiltrating lymphocytes derived from the spontaneous ovarian tumors following intra-peritoneal injection of chimeric NKG2D-Fc-RO protein. 10-week-old tumor-bearing MISIIR mice were treated with $20 \mu \mathrm{g}$ /mouse of NKG2D-Fc or NKG2D-Fc-RO protein every week for four weeks and $2.5 \times 10^{6}$ OT -1 CD $8+T$ cells were injected intraperitoneally every other week two times. Mice were sacrificed 1 week after the last treatment. Ovarian tumors were harvested and tumor infiltrating lymphocytes were characterized for OVA-specific CD8+ T cells using OVA peptide-loaded $\mathrm{H}-2 \mathrm{~K}^{\mathrm{b}}$ tetramer staining. A. Representative flow cytometry analysis for the characterization of OVA-specific CD8+ T cells in the tumors. B. Bar graph depicting the percentage of OVA-specific CD8+ T cells in total TILs (mean \pm SD). 
are capable of specifically targeting NKG2D ligands, often found to be overexpressed on tumor cells, making the NKG2D-ligand system an exploitable receptor-ligand system to bring potentially immune-modulating or therapeutic molecules to the tumor site.

The data generated from our current study is consistent with data generated from previous studies using NKG2D as a carrier to deliver protein/antigen of interest to NKG2D ligand expressing tumor cells in tumor bearing mice for the development of therapeutic approaches. Previously, we used NKG2D to specifically deliver IL-2 to Rae-1 expressing TC-1 tumors in tumor-bearing mice. We found that by linking NKG2D to IL2, we were able to specifically deliver IL-2 to the tumor location, enhancing antigen-specific Tcell immune response and controlling tumor growth [15].

Our approach successfully exploited an NKG2D ligand expressed on tumor cells, to specifically deliver a therapeutic agent. Our findings warrant the examination of other molecules that are also uniquely expressed or overexpressed on the surface of tumor cells, but not on normal cells. For example, mesothelin is a commonly overexpressed protein on many human cancer cells, including ovarian [5,6] and pancreatic cancer [5]. Indeed, we have previously utilized mesothelin for targeting ovarian tumor cells [4]. Other conceivable molecules for targeting ovarian cancer cells include follicle-stimulating hormone receptor (FSHR) [17], intercellular adhesion molecule 1 (ICAM-1) [18], Müllerian inhibiting substance type II receptor (MISIIR) [19], and human epidermal growth factor receptor 2 (HER2) [20]. Leading-edge technologies including deep sequencing, gene and protein microarrays, proteomics, have the capacity to identify more potential candidate targets in the near future that may be used to deliver specific molecules to the tumor loci using a receptor-ligand system or an antibody-antigen system.

One potential limitation for the clinical translation of the proposed approach is antibody generation, specifically against the chimeric protein. If antibodies are generated against the chimeric protein, subsequent challenges may be affected. Furthermore, there are concerns that the chimeric protein may be allergenic and could be rapidly eliminated due to immune responses against the chimeric protein. Therefore, as a step towards clinical translation, it will be important to use the human counterparts for the murine NKG2D, Fc, R, and $\mathrm{O}$ of this study to create a clinical grade reagent that elicits limited immune responses against the therapeutic chimeric protein. It will also be important to test the binding ability of the human NKG2D-Fc-RO to various human ovarian cancer cell lines before considering generating a clinical grade reagent for clinical trials.

\section{Conclusions}

In summary we have successfully developed a strategy to specifically target a therapeutic chimeric protein to tumor loci, which elicits potent tumor-targeted killing through antigen-specific CD8+ immune responses. This strategy may provide a platform for the delivery various anti-cancer molecules to the tumor loci as well as for coating tumor cells to circumvent immune tolerance in order to generate therapeutic antitumor effects. Furthermore, this strategy can be used in conjunction with active immunization against foreign antigenic peptide that is incorporated in the chimeric protein in order to enhance the antigenspecific CD8+ T cell immune responses. Since NKG2D ligands are highly expressed in many different tumor cells, our strategy represents a potentially useful approach for delivering molecules of interest to tumor loci for the control of different kinds of tumors for future clinical translation.

\section{Methods}

Mice

Female TgMISIIR-TAg transgenic mice were obtained by breeding female C57BL/6 mice with male TgMISIIRTAg transgenic mice [21]. The male MISIIR-TAg transgenic mice were obtained from Fox Chase Cancer Center (Philadelphia, PA, USA) [21]. Female TgMISIIR-TAg transgenic mice 10 weeks of age were used in the experiments. These mice spontaneously developed ovarian tumors with complete tissue penetration. All animals were maintained under specific pathogen-free conditions, and all procedures were performed according to approved protocols and in accordance with recommendations for the proper use and care of laboratory animals by Johns Hopkins University Animal Care and Use Committee (protocol MO11M398).

\section{Cells}

The MOVCAR (mouse ovarian carcinoma) cell line was obtained from Fox Chase Cancer Center (Philadelphia, PA, USA). It was derived from ascites of a TgMISIIR-TAg transgenic mouse with ovarian tumors [22]. All cell lines were maintained in RPMI 1640 complete medium supplemented with $10 \%$ heat-inactivated FBS (HyClone), 1\% non-essential amino acid, $2 \mathrm{mM}$ l-glutamine, $50 \mathrm{U} / \mathrm{ml}$ penicillin, $50 \mathrm{mg} / \mathrm{ml}$ streptomycin, and $5 \mathrm{mM}$ 2-ME (Invitrogen Life Technologies). To generate the MOVCAR-luc cell line, luciferase expressing MOVCAR tumor cells (MOVCAR-luc) were generated by transducing the MOVCAR cells with retrovirus containing luciferase, pLuci-thy1.1, and flow cytometry sorting following the previously described protocol [23]. OVA-specific CD8+ T cells (OT-1) were produced via the stimulation of splenocytes obtained from OT-1 transgenic mice with irradiated EG.7 cells in the presence of interleukin-2 (20 IU/ml, Pepro-Tech, Rock Hill, NJ).

\section{Plasmid DNA constructs and preparation}

Pfuse-NKG2D-Fc was described previously [15]. To generate pFuse-NKG2D-Fc-RO the oligos CTAGACGGGT 
GAAGCGGAGTATAATCAACTTTGAAAAACTGTAAC and TCGAGTTACAGTTTTTCAAAGTTGATTATACTC CGCTTCACCCGT were annealed and cloned into XbaI/ Xho sites of Pfuse-NKG2D-Fc.

\section{Transfection and protein purification}

For purification of the NKG2D-FC and NKG2D-FC-RO, $50 \mu \mathrm{g}$ of plasmid was transfected into $1 \times 10^{7} \mathrm{FD} 11$ cells in T-150 flask using Lipofectamin 2000 (Invitrogen Corp., Carlsbad, CA, USA). After 3 days, cell culture media was accumulated, filtered using $0.22 \mu \mathrm{m}$ syringe filter (Millipore, Billerica, MA, USA) and concentrated with Amicon cut-off 50 kDa Ultra -15 (Millipore, Billerica, MA, USA). Concentrated recombinant protein containing media was applied to a HiTrap Protein G HP column (GE Healthcare) following the vendor's protocol. Protein concentrations were determined by the Coomassie Plus protein assay (Pierce, Rockford, IL, USA) and purity was estimated by SDS polyacrylamide gel electrophoresis.

\section{Cell staining and flow cytometry analysis}

For flow cytometry analysis, tumor cells were stained with Rae-1 antibody (BD Bioscience), or $0.5 \mu \mathrm{g}$ of NKG2D-Fc and NKG2D-Fc-RO respectively and PE-conjugated antimouse antibody was used as a detection antibody (BD Bioscience). The percentage of OVA-specific IFN- $\gamma$ secreting $\mathrm{CD}^{+} \mathrm{T}$ cells was determined using intracellular cytokine staining and FACScan analysis with CELLQuest software (Becton Dickinson Immunocytometry System, Mountain View, USA).

OVA-specific T cell activation and In vitro cytotoxicity assay For $\mathrm{T}$ cell activation, MOVCAR cells were added to 48well plates at a dose of $1 \times 10^{5}$ cells/well and incubated with $0.5 \mu \mathrm{g} / \mathrm{ml}$ of proteins. Eighteen hours later, treated tumor cells were incubated with $2 \times 10^{5}$ OVA-specific cytotoxic $\mathrm{T}$ cells (CTL). One day after activation, IFN$\gamma$-secreting OVA-specific CTLs were identified by intracellular cytokine staining and analyzed by flow cytometry analysis. For the in vitro cytotoxicity experiment, $1 \times 10^{4}$ of luciferase-expressing MOVCAR (MOCAR-luc) cells were incubated with $0.5 \mu \mathrm{g} / \mathrm{ml}$ of one of the various proteins on 96-well plate for 6 hours and treated with $2 \times 10^{4}$ OVA-specific CTLs. The degree of CTL-mediated killing of the tumor cells was measured by the IVIS luminescence imaging system series 2000 as described previously [4].

\section{In vivo tumor treatment experiments}

Tumor growth was assessed in 10-week-old female TgMISIIR-TAg transgenic mice by visual inspection following open surgery. Mice (5 per group) with similar sized ovarian tumors were selected to receive treatment with $20 \mu \mathrm{g} /$ mouse of NKG2D-Fc or NKG2D-Fc-RO protein every week for four weeks. Prior to treatment, mice to be treated with NKG2D-Fc had an average tumor size of $55.02 \pm 21.18 \mathrm{~mm}^{3}$ and mice to be treated with NKG2D-Fc-RO had an average tumor size of $52.36 \pm 27.04 \mathrm{~mm}^{3}$. Mice were intraperitoneally injected with $2.5 \times 10^{6}$ OT-1 CD8+ T cells twice, every other week. Female TgMISIIR-TAg transgenic mice without treatment were also included for comparison. Mice were sacrificed 1 week after the last treatment. Ovarian tumors were harvested for measurement. Tumor infiltrating lymphocytes (TILs) in the ovarian tumors were characterized for the presence of OVA-specific CD8+ T cells using OVA peptide-loaded $\mathrm{H}-2 \mathrm{~K}^{\mathrm{b}}$ tetramer staining and CD8 staining.

\section{Statistical analysis}

The data presented in this study are representative of at least two experiments performed, and are expressed as means \pm standard deviation (S.D.). The number of samples in each group for any given experiment was $>3$. Results for intracellular cytokine staining with flow cytometry analysis and tumor treatment experiments were evaluated by analysis of variance (one-way ANOVA) and the TukeyKramer multiple comparison test. Comparisons between individual data points were performed using Student's ttest. Statistical analysis was performed with GraphPad Prism 4.0 software and a $\mathrm{p}$ value $<0.05$ was considered significant.

\begin{abstract}
Abbreviations
R: Furin cleavage site; CTL: Cytotoxic T lymphocyte; Meso: Mesothelin; SCFv: Single chain variable fragment; OVA: O - ovalbumin; MHC: Major histocompatibility complex; MOVCAR: Mouse ovarian carcinoma cell line; OT-1: OVA-specific CD8+ T cells; MISIIIR: Müllerian inhibiting substance type ॥ receptor; FSHR: Follicle-stimulating hormone receptor; ICAM-1: Intercellular adhesion molecule 1; HER2: Human epidermal growth factor receptor 2.
\end{abstract}

\section{Competing interests}

The authors declare that they have no competing interests.

\section{Authors' contributions}

THK, CFH and TCW conceived and designed experiments and interpreted data. THK, YCT and LH performed experiments. JK, BY, CFH and TCW wrote the manuscript. All authors read and approved the final manuscript.

\section{Acknowledgements}

This work was supported by the American Cancer Society.

\section{Author details}

${ }^{1}$ Department of Immunology, College of Medicine, Konkuk University, Chungju, South Korea. ${ }^{2}$ Department of Pathology, School of Medicine, Johns Hopkins University, Bldg. CRBII Rm. 309, 1550 Orleans Street, Baltimore, Maryland 21231, USA. ${ }^{3}$ Department of Obstetrics and Gynecology, Johns Hopkins University, Baltimore, MD 21205, USA. ${ }^{4}$ Department of Molecular Microbiology and Immunology, Johns Hopkins University, Baltimore, MD 21205, USA. ${ }^{5}$ Department of Oncology, Johns Hopkins University, Baltimore, MD 21205, USA.

Received: 4 November 2013 Accepted: 14 November 2013 Published: 20 December 2013

\section{References}

1. Baum M, Ebb S, Brooks M: Biological fallout from trials of adjuvant tamoxifen in early ovarian cancer. Adjuvant Ther Cancer 1990, 6:269-274. 
2. Swain SM: Selection of therapy for stage III breast cancer. Surg Clin North Am 1990, 70:1061-1080.

3. Sznol M, Holmlund J: Antigen-specific agents in development. Semin Oncol 1997, 24:173-186.

4. Kang TH, Ma B, Wang C, Wu TC, Hung CF: Targeted coating with antigenic peptide renders tumor cells susceptible to CD8(+) T cell-mediated killing Mol Ther: J Am Soc Gene Ther 2013, 21:542-553.

5. Scholler N, Fu N, Yang Y, Ye Z, Goodman GE, Hellstrom KE, Hellstrom I: Soluble member(s) of the mesothelin/megakaryocyte potentiating factor family are detectable in sera from patients with ovarian carcinoma. Proc Natl Acad Sci U S A 1999, 96:11531-11536.

6. Hassan R, Bera T, Pastan I: Mesothelin: a new target for immunotherapy. Clin Cancer Res 2004, 10:3937-3942.

7. Coppola JM, Bhojani MS, Ross BD, Rehemtulla A: A small-molecule furin inhibitor inhibits cancer cell motility and invasiveness. Neoplasia 2008, 10:363-370.

8. Page RE, Klein-Szanto AJ, Litwin S, Nicolas E, Al-Jumaily R, Alexander P, Godwin AK, Ross EA, Schilder RJ, Bassi DE: Increased expression of the pro-protein convertase furin predicts decreased survival in ovarian cancer. Cell Oncol 2007, 29:289-299.

9. Mercapide J, Lopez De Cicco R, Bassi DE, Castresana JS, Thomas G, Klein-Szanto $\mathrm{AJ}$ : Inhibition of furin-mediated processing results in suppression of astrocytoma cell growth and invasiveness. Clin Cancer Res 2002, 8:1740-1746.

10. Bassi DE, Mahloogi H, Al-Saleem L, Lopez De Cicco R, Ridge JA, Klein-Szanto AJ: Elevated furin expression in aggressive human head and neck tumors and tumor cell lines. Mol Carcinog 2001, 31:224-232.

11. Cheng M, Watson PH, Paterson JA, Seidah N, Chretien M, Shiu RP: Pro-protein convertase gene expression in human breast cancer. Int J Cancer 1997, 71:966-971.

12. Schalken JA, Roebroek AJ, Oomen PP, Wagenaar SS, Debruyne FM, Bloemers HP, Van de Ven WJ: Fur gene expression as a discriminating marker for small cell and nonsmall cell lung carcinomas. J Clin Invest 1987, 80:1545-1549.

13. Raulet DH: Roles of the NKG2D immunoreceptor and its ligands. Nat Rev Immun 2003, 3:781-790.

14. Nausch N, Cerwenka A: NKG2D ligands in tumor immunity. Oncogene 2008, 27:5944-5958

15. Kang TH, Mao CP, He L, Tsai YC, Liu K, La V, Wu TC, Hung CF: Tumor-targeted delivery of IL-2 by NKG2D leads to accumulation of antigen-specific CD8+ T cells in the tumor loci and enhanced anti-tumor effects. PloS One 2012, 7:e35141.

16. Zhang T, Lemoi BA, Sentman CL: Chimeric NK-receptor-bearing T cells mediate antitumor immunotherapy. Blood 2005, 106:1544-1551.

17. Choi $\mathrm{JH}$, Choi KC, Auersperg N, Leung PCK: Overexpression of follicle-stimulating hormone receptor activates oncogenic pathways in preneoplastic ovarian surface epithelial cells. J Clin Endocrinol Metab 2004, 89:5508-5516.

18. Ramakrishna V, Negri DRM, Brusic V, Fontanelli R, Canevari S, Bolis G, Castelli C, Parmiani G: Generation and phenotypic characterization of new human ovarian cancer cell lines with the identification of antigens potentially recognizable by HLA,Äêrestricted cytotoxic T cells. Int I Cancer 1997, 73:143-150.

19. Bakkum-Gamez JN, Aletti G, Lewis KA, Keeney GL, Thomas BM, Navarro-Teulon I, Cliby WA: Müllerian inhibiting substance type II receptor (MISIIR): a novel, tissue-specific target expressed by gynecologic cancers. Gynecol Oncol 2008, 108:141-148.

20. Durrant LG, Pudney VA, Spendlove I: Using monoclonal antibodies to stimulate antitumor cellular immunity. Expert Rev Vaccines 2011, 10:1093-1106.

21. Connolly DC, Bao R, Nikitin AY, Stephens KC, Poole TW, Hua X, Harris SS, Vanderhyden BC, Hamilton TC: Female mice chimeric for expression of the simian virus $40 \mathrm{TAg}$ under control of the MISIIR promoter develop epithelial ovarian cancer. Cancer Res 2003, 63:1389-1397.

22. Quinn BA, Xiao F, Bickel L, Martin L, Hua X, Klein-Szanto A, Connolly DC. Development of a syngeneic mouse model of epithelial ovarian cancer. J Ovarian Res 2010, 3:24.

23. Hung CF, Tsai YC, He L, Wu TC: Control of mesothelin-expressing ovarian cancer using adoptive transfer of mesothelin peptide-specific CD8+ $T$ cells. Gene Ther 2007, 14:921-929.

doi:10.1186/2045-3701-3-48

Cite this article as: Kang et al:: Control of spontaneous ovarian tumors by CD8+ T cells through NKG2D-targeted delivery of antigenic peptide. Cell \& Bioscience 2013 3:48.

\section{Submit your next manuscript to BioMed Central and take full advantage of:}

- Convenient online submission

- Thorough peer review

- No space constraints or color figure charges

- Immediate publication on acceptance

- Inclusion in PubMed, CAS, Scopus and Google Scholar

- Research which is freely available for redistribution 